\title{
Left Ventricular Global Strain for Estimating Relaxation and Filling Pressure
}

\author{
- A Multicenter Study -
}

Taichi Hayashi, MD; Satoshi Yamada, MD, PhD; Hiroyuki Iwano, MD, PhD;

Masahiro Nakabachi, BSc; Mamoru Sakakibara, MD, PhD; Kazunori Okada, PhD;

Daisuke Murai, MD; Hisao Nishino, BSc; Kenya Kusunose, MD, PhD;

Kiyotaka Watanabe, MD, PhD; Tomoko Ishizu, MD, PhD; Kazuaki Wakami, MD, PhD;

Hirotsugu Yamada, MD, PhD; Kaoru Dohi, MD, PhD; Yoshihiro Seo, MD, PhD;

Nobuyuki Ohte, MD, PhD; Taisei Mikami, MD, PhD; Hiroyuki Tsutsui, MD, PhD

\begin{abstract}
Background: Speckle-tracking echocardiography (STE)-derived parameters may have better correlation with left ventricular (LV) relaxation and filling pressure than tissue Doppler-derived parameters. However, it has not been elucidated which parameter - strain or strain rate - and which direction of myocardial deformation - longitudinal or circumferential - is the most useful marker of LV relaxation and filling pressure.
\end{abstract}

Methods and Results: We conducted a prospective multicenter study and compared the correlation of tissue Doppler- and STE-derived parameters with the time constant of LV pressure decay $(\tau)$ and LV mean diastolic pressure (MDP) in 77 patients. The correlation of early-diastolic mitral annular velocity (e') with $\tau$ was weak $(r=-0.32$, $P<0.01)$, and that of peak longitudinal strain (LS) was the strongest $(r=-0.45, P<0.001)$ among the STE-derived parameters. There was a modest correlation between LVMDP and the ratio of early-diastolic inflow velocity $(E)$ to $e^{\prime}$ $\left(E / e^{\prime}\right)(r=0.50, P<0.001)$. In contrast, the ratio of $E$ to LS (E/LS) correlated strongly with LVMDP $(r=0.70, P<0.001)$. The correlation of E/LS with LVMDP was significantly better than that for $E / e^{\prime}(P<0.01)$. Receiver-operating characteristic analysis showed that E/LS had the largest area under the curve for distinguishing elevated LVMDP (E/LS 0.86, E/e' 0.74, E/A 0.67).

Conclusions: STE-derived longitudinal parameters correlated well with LV relaxation and filling pressure. In particular, E/LS could be more accurate than E/e' for estimating LV filling pressure. (Circ J 2016; 80: 1163-1170)

Key Words: Diastolic dysfunction; Heart diseases; Heart failure; Speckle-tracking echocardiography

$\mathbf{E}$ levated left ventricular (LV) filling pressure results in shortness of breath, which is both a major symptom and a prognostic predictor for heart failure. Therefore, noninvasive estimation of LV filling pressure is considered a goal of routine echocardiography. Recent recommendations encourage using tissue Doppler-derived early-diastolic mitral annular velocity ( $\left.\mathrm{e}^{\prime}\right)$ as a parameter of $\mathrm{LV}$ relaxation and the ratio of early-diastolic LV inflow velocity (E) to $e^{\prime}\left(E / e^{\prime}\right)$ to indicate LV filling pressure. ${ }^{1}$ However, recent reports suggest that these parameters do not necessarily correlate with invasively measured LV filling pressure in patients with hypertrophic cardiomyopathy, ${ }^{2}$ severely reduced LV systolic function, ${ }^{3}$ and heart failure with preserved ejection fraction $(\mathrm{EF}){ }^{4}$

\section{Editorial p1104}

Reasons for inaccuracies with tissue Doppler-derived parameters could include angle dependency and myocardial tethering,

Received February 1, 2016; revised manuscript received February 17, 2016; accepted February 22, 2016; released online March 25, 2016 Time for primary review: 8 days

Department of Cardiovascular Medicine, Hokkaido University Graduate School of Medicine, Sapporo (T.H., S.Y., H.I., M.S., D.M., H.T.); Division of Laboratory and Transfusion Medicine, Hokkaido University Hospital, Sapporo (M.N., H.N.); Faculty of Health Sciences, Hokkaido University, Sapporo (K.O., T.M.); Department of Cardiovascular Medicine, Tokushima University Hospital, Tokushima (K.K., H.Y.); Department of Cardiology and Nephrology, Mie University Graduate School of Medicine, Tsu (K. Watanabe, K.D.); Cardiovascular Division, Faculty of Medicine, University of Tsukuba, Tsukuba (T.I., Y.S.); Department of Cardio-Renal Medicine and Hypertension, Nagoya City University Graduate School of Medical Sciences, Nagoya (K. Wakami, N.O.), Japan

Mailing address: Satoshi Yamada, MD, PhD, Department of Cardiovascular Medicine, Hokkaido University Graduate School of Medicine, Kita-15, Nishi-7, Kita-ku, Sapporo 060-8638, Japan. E-mail: syamada@med.hokudai.ac.jp

ISSN-1346-9843 doi:10.1253/circj.CJ-16-0106

All rights are reserved to the Japanese Circulation Society. For permissions, please e-mail: cj@j-circ.or.jp 


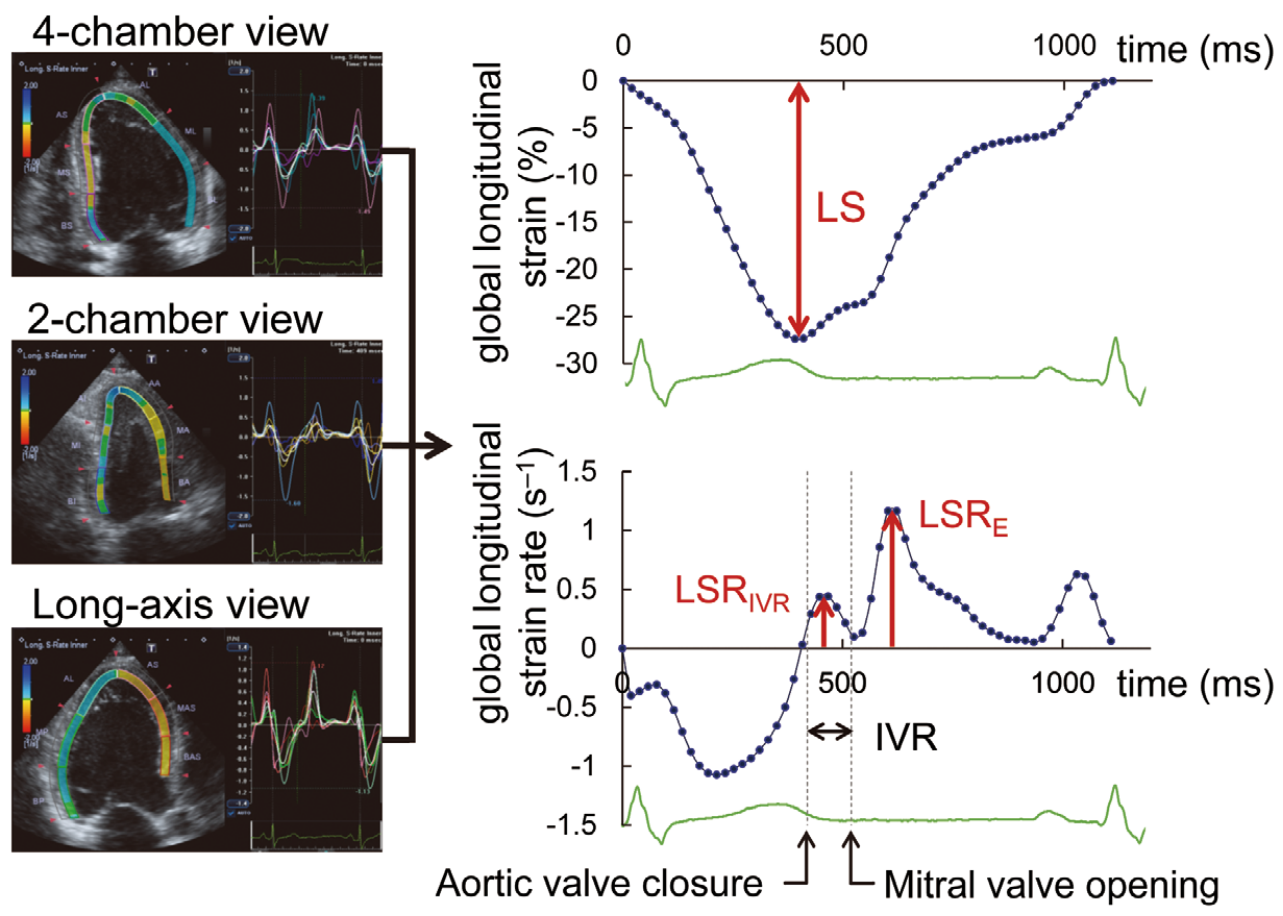

Figure 1. Examples of time-global longitudinal strain and strain rate curves. IVR, isovolumic relaxation; LS, absolute value of peak longitudinal strain; LSRIVR, peak longitudinal strain rate during the IVR period; LSRE, peak longitudinal strain rate in the early-diastolic phase.

which are well-known limitations of tissue Doppler imaging. ${ }^{5}$ Because $\mathrm{e}^{\prime}$ reflects the rate of longitudinal LV wall expansion in early diastole, a deformation parameter for the entire LV wall could be an alternative or even more useful marker of LV relaxation. Based on this hypothesis, myocardial strain and strain rate measured by 2-dimensional (2D) speckle-tracking echocardiography (STE) have been tested as estimates of LV relaxation and filling pressure. ${ }^{5-11}$ Several single-center studies have demonstrated that STE-derived parameters correlate with LV relaxation and filling pressure better than tissue Dopplerderived parameters. ${ }^{5-10}$ However, it has not been elucidated which parameter - peak strain, strain rate during the isovolumic relaxation (IVR) period or during the early-diastolic phase - and which direction of the myocardial deformation - longitudinal or circumferential - are the most useful markers of LV relaxation and filling pressure. Accordingly, we conducted a

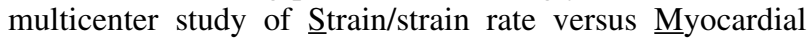
velocity for Assessing left ventricular relaxation and filling Pressure (SMAP study). The aims of this study were to (1) re-examine the correlations between invasively measured LV hemodynamic parameters and tissue Doppler-derived parameters and (2) determine the most useful marker(s) of LV relaxation and filling pressure among STE-derived parameters.

\section{Methods}

\section{Study Population and Protocol}

We prospectively enrolled 84 patients who underwent cardiac catheterization for clinical diagnosis of cardiac diseases, including angina pectoris, prior myocardial infarction, dilated cardiomyopathy, hypertrophic cardiomyopathy, and hyperten- sive heart disease, from 5 university hospitals in Japan (Hokkaido University Hospital, Nagoya City University Hospital, Mie University Hospital, University of Tsukuba Hospital, and Tokushima University Hospital). We excluded patients with atrial fibrillation, frequent supraventricular or ventricular premature contraction, moderate to severe aortic or mitral valve disease, and inadequate echocardiographic images. Patients who had undergone mitral valve plasty, replacement, or aortic valve replacement, and those who underwent hemodialysis were also excluded. Echocardiography was performed within $3 \mathrm{~h}$ of cardiac catheterization. The study protocol was approved by the institutional review board of each hospital and all patients gave written informed consent.

\section{Pressure Measurement}

LV pressures were recorded prior to coronary angiography. A high-fidelity, 5-French (Model SPC-454D, Millar Instruments, Houston, TX, USA) or 6-French (Model CA-61000-PLB, CD Leycom, Zoetermeer, The Netherlands) pigtail micromanometer-tipped catheter was balanced to the zero level at the midaxillary line. The catheter was then placed in the LV chamber via the femoral or brachial artery and calibrated against the pressure measured simultaneously with the fluid-filled lumen at end diastole. $\mathrm{LV}$ pressure was recorded at end expiration for 3 consecutive cardiac cycles and digitized with a 1-ms sampling frequency using a data analysis system (PowerLab and LabChart software version 7.3.4, ADInstruments, NSW, Australia). The time constant of LV pressure decay $(\tau)$ was derived from a monoexponential fit to the LV pressure decay with a zero asymptote. ${ }^{12}$ The $\tau$ with the zero-asymptote method may be less sensitive for the detection of impaired LV 
relaxation than that with a non-zero-asymptote method; however, many clinical studies and the European guidelines for the diagnosis of heart failure with preserved $\mathrm{EF}^{13}$ have used the $\tau$ derived from the zero-asymptote method. We thus followed the prior standards in the present study. LV mean diastolic pressure (LVMDP) was measured as previously described. ${ }^{14}$ Abnormal LV relaxation was defined as $\tau \geq 48 \mathrm{~ms}$ and elevated LV filling pressure was defined as LVMDP $\geq 12 \mathrm{mmHg} .{ }^{15}$ All parameters were averaged over 3 consecutive cardiac cycles.

\section{Echocardiography}

Echocardiography was performed using 1 of 2 commercially available systems (Aplio Artida SSH-88-CV or Aplio SSA770A, Toshiba Medical Systems, Tochigi, Japan). LV mass was calculated according to the recommendations. ${ }^{16} \mathrm{LV}$ hypertrophy was defined as LV mass index $\geq 115 \mathrm{~g} / \mathrm{m}^{2}$ in males and $\geq 95 \mathrm{~g} / \mathrm{m}^{2}$ in females. LVEF was calculated using the biplane method of disks. Patients were divided into 2 groups: with reduced $\mathrm{LVEF}(<50 \%)$ and with preserved LVEF $(\geq 50 \%)$. LV inflow $\mathrm{E}$ and atrial-systolic peak velocity (A) were measured using pulsed-wave Doppler. Using the apical 4-chamber view, $\mathrm{e}^{\prime}$ was measured at the septal and lateral sides of the annulus and averaged. The IVR period was defined as the time from aortic valve closure, determined by the end of LV outflow on the pulsed-Doppler image, to mitral valve opening, determined by the beginning of LV inflow.

\section{STE Analysis}

LV myocardial strain was analyzed offline using STE software (Toshiba Medical Systems). First, the endocardial border was manually traced, and the myocardial motion was tracked frame-by-frame on every view. The longitudinal strain/strain rate were measured in the apical 4-chamber, 2-chamber, and long-axis views and averaged frame-by-frame to construct a time-global strain curve as well as a time-global strain rate curve (Figure 1). Next, the absolute value of peak longitudinal strain (LS), peak longitudinal strain rate in the early-diastolic phase (LSRE), and during the IVR period (LSRIVR) in the inner layer were measured (Figure 1). The ratios of $E$ to LS (E/LS), E to LSRE (E/LSRE), and E to LSRIVR (E/LSRIVR) were calculated. Similarly, circumferential strain and strain rate were measured in the short-axis views at the basal, middle, and apical ventricular levels. The absolute value of peak circumferential strain (CS), peak circumferential strain rate in the early-diastolic phase (CSRE) and during the IVR (CSRIVR) in the inner layer were measured, and the ratios of $\mathrm{E}$ to $\mathrm{CS}(\mathrm{E} /$ $\mathrm{CS}), \mathrm{E}$ to $\mathrm{CSRE}$ (E/CSRE), and E to CSRIVR (E/CSRIVR) were calculated.

\section{Data Collection}

The study protocol was written by the investigators from Hokkaido University (S.Y. and H.I.), and reviewed and modified by the members of the steering committee (S.Y., H.Y., K.D., Y.S., and N.O.), including an echocardiographic measurement manual that was followed by each institution to ensure consistent measurements. Echocardiographic tracking was performed in each institution and all STE data were exported to a spreadsheet. The data sets including clinical data, LV pressure measurements, conventional echocardiographic measurements, and the STE analysis spreadsheets were sent to the investigators at Hokkaido University for analysis (T.H., S.Y. H.I., and M.N.).

\section{Statistical Analysis}

Data are presented as mean \pm standard deviation. Liner regres-

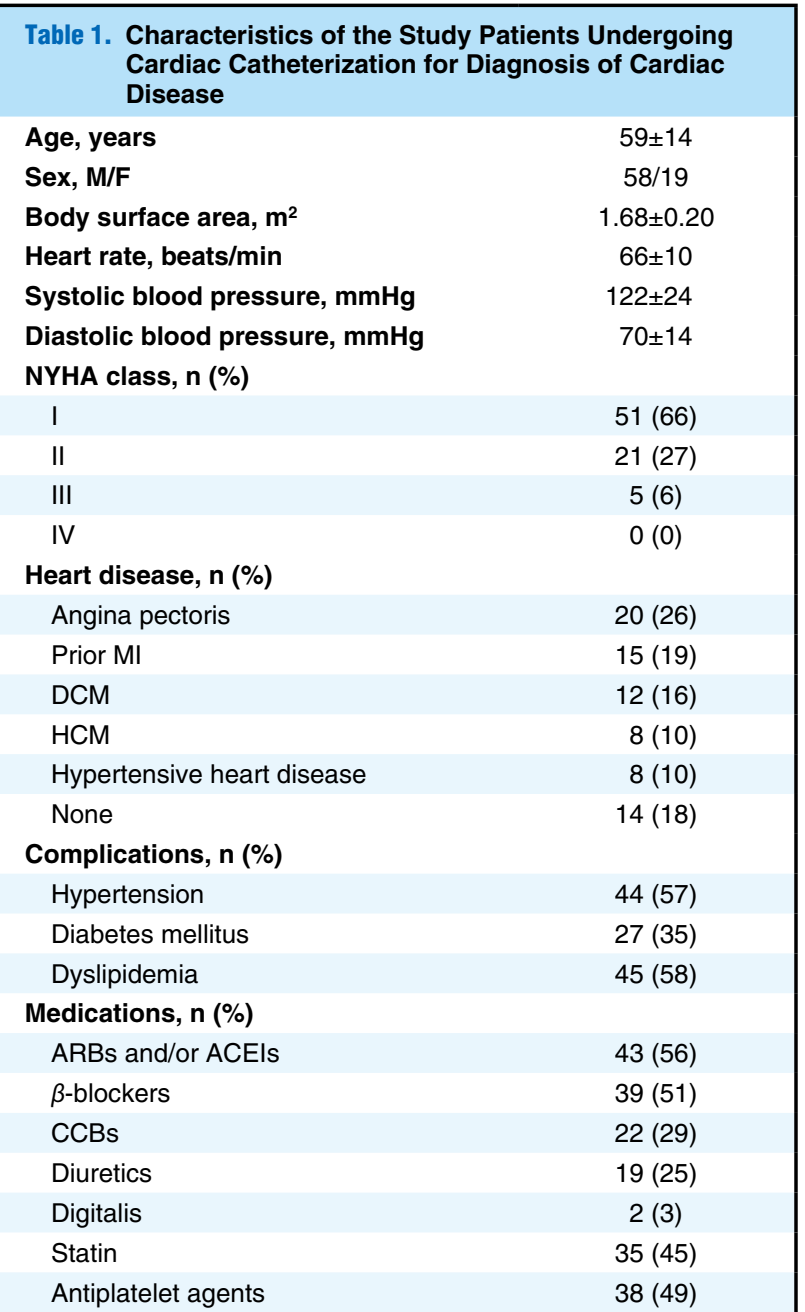

ACEI, angiotensin-converting enzyme inhibitor; ARB, angiotensin II receptor-blocker; CCB, calcium-channel blocker; DCM, dilated cardiomyopathy; HCM, hypertrophic cardiomyopathy; MI, myocardial infarction; NYHA, New York Heart Association.

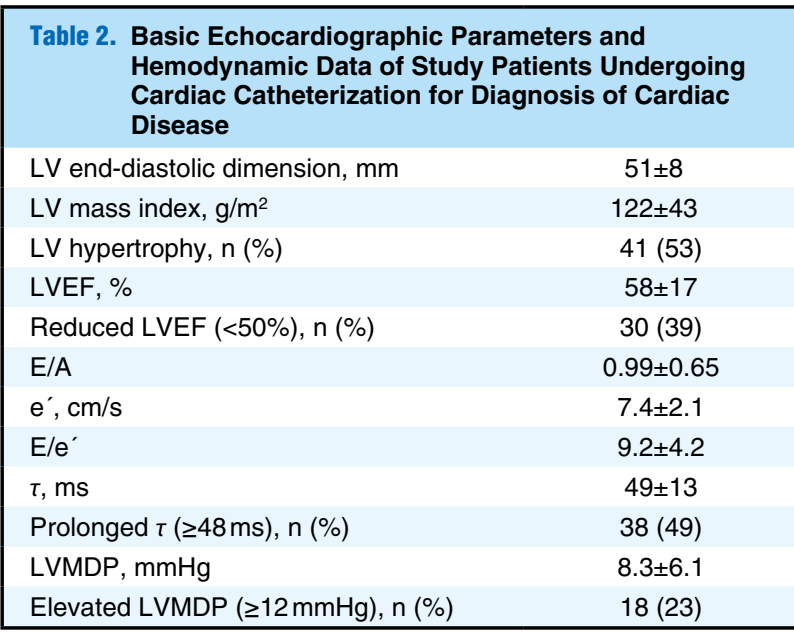

$E / A$, ratio of early-diastolic LV inflow velocity $(E)$ to atrial-systolic velocity (A); $e^{\prime}$, early-diastolic mitral annular velocity; $E / e^{\prime}$, ratio of $E$ to $e^{\prime} ; E F$, ejection fraction; LV, left ventricular; MDP, mean diastolic pressure. 

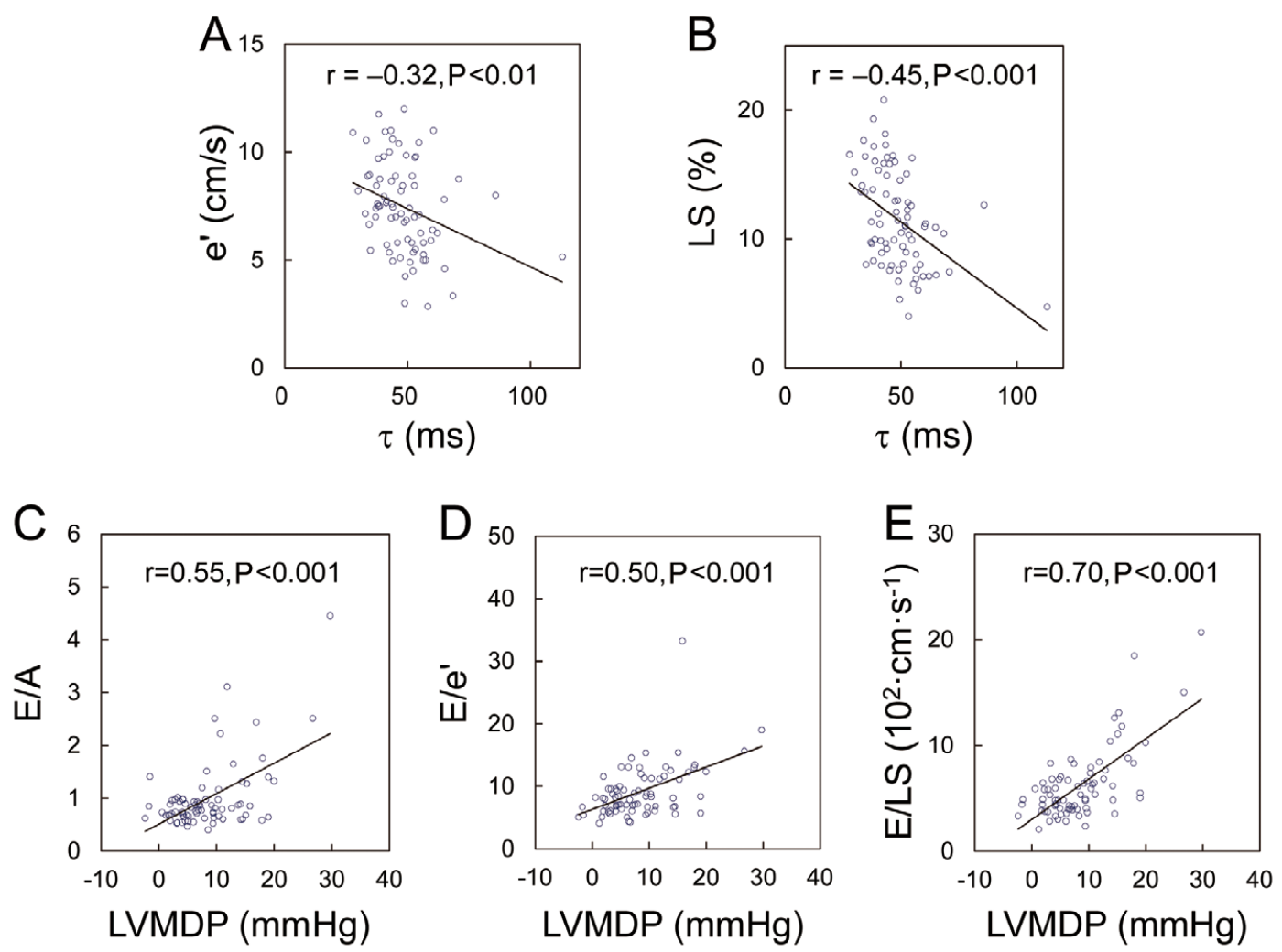

Figure 2. Correlations of echocardiographic parameters with $\tau$ and left ventricular mean diastolic pressure (LVMDP). E/A, ratio of early-diastolic LV inflow velocity (E) to atrial-systolic velocity (A); e', early-diastolic mitral annular velocity; E/e', ratio of E to e'; E/ $L S$, ratio of $E$ to LS; LS, absolute value of peak longitudinal strain.

sion analysis was performed to determine the correlation between parameters. Two correlation coefficients drawn from the same sample were compared following Cohen et al's method. ${ }^{17}$ In patients who had a peak strain rate during the IVR period (SRIVR) of approximately zero, E/SRIVR was corrected to a normal distribution. We set the upper limit of 1/ SRIVR as the 3rd quartile plus $1.5 \times$ (interquartile range): $20 \mathrm{~s}$ in 1/LSRIVR and $6.7 \mathrm{~s}$ in 1/CSRIVR. We regarded 1/LSRIVR as $20 \mathrm{~s}$ and $1 / \mathrm{CSR}_{\mathrm{IVR}}$ as $6.7 \mathrm{~s}$ in patients with $1 / \mathrm{SR}$ IVR over the limit. To assess the diagnostic ability of the echocardiographic parameters, we performed receiver-operating characteristic (ROC) analysis. Significant differences between the areas under the ROC curves were tested using Hanley and McNeil's method. ${ }^{18}$ Also, to investigate the incremental diagnostic value of the parameters, we conducted interactive stepwise procedures. The incremental value of the added variables was determined by comparing the global chi-square value, which was calculated from the log likelihood ratio. The reproducibility of the STE analysis was assessed in 17 patients; 2 independent, blinded observers analyzed the same $2 \mathrm{D}$ cine-loops and 1 of them repeated the analysis on a separate day. SPSS Statistics software (version 19.0.0, SPSS Inc, Chicago, IL, USA), R software (version 3.0.1, R Foundation for Statistical Computing, Vienna, Austria), and JMP software (version 11.0.0, SAS Institute Inc, Cary, NC, USA) were used for the statistical calculations. The results were considered statistically significant with $\mathrm{P}<0.05$.

\section{Results}

Of the 84 patients who were eligible for study enrollment, 1 patient was excluded from the study because atrial fibrillation occurred during catheterization, 1 patient because of inability to measure LV pressure, 3 patients because of moderate mitral regurgitation, and 2 patients because of inadequate $\mathrm{LV}$ wall tracking in the STE analysis. All data from the remaining 77 patients were analyzed in this study.

\section{Patients' Characteristics}

Patients' characteristics are summarized in Table 1. Of 34 patients who were suspected of having angina pectoris, 20 were diagnosed as having angina pectoris by coronary angiography. In the remaining 14 patients, coronary angiography and echocardiography revealed no coronary artery disease or other cardiac diseases. Echocardiographic and hemodynamic parameters are presented in Table 2; 30 patients (39\%) had reduced LVEF and 47 (61\%) had preserved LVEF; 38 patients (49\%) had abnormal LV relaxation and $18(23 \%)$ had elevated LV filling pressure.

\section{Correlations Between $\tau$ and Echocardiographic Parameters}

There was a weak, but significant, correlation between $\tau$ and $\mathrm{e}^{\prime}$ (Figure 2A). The correlations between $\tau$ and the STEderived parameters are summarized in Table 3 . There were weak correlations between $\tau$ and the STE-derived parameters 
Table 3. STE-Derived Parameters and Their Correlations With $\tau$ and LVMDP in Study Patients Undergoing Cardiac Catheterization for Diagnosis of Cardiac Disease

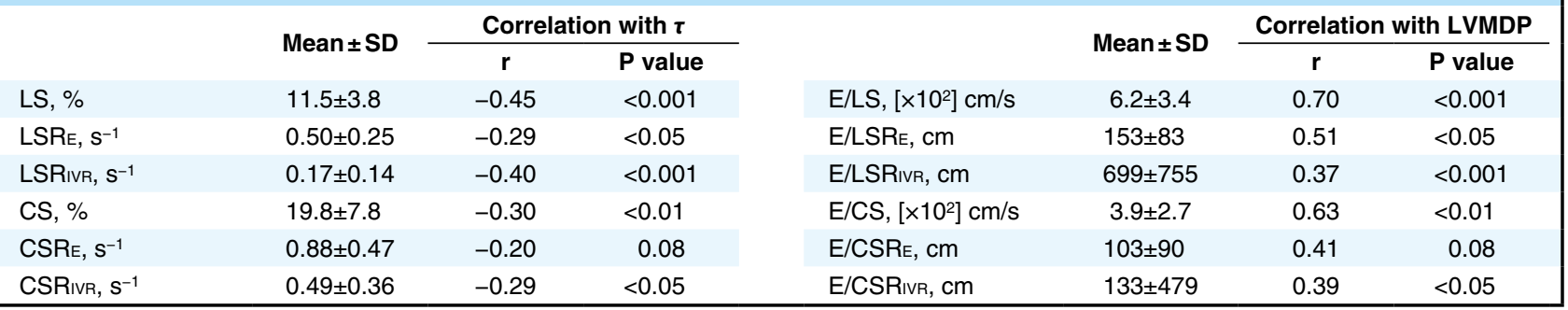

CS, absolute value of peak circumferential strain; CSRE, peak circumferential strain rate in the early-diastolic phase; CSRIVR, peak circumferential strain rate during the IVR period; $E / C S$, ratio of $E$ to CS; E/CSRE, ratio of E to CSRE; E/CSRIVR, ratio of E to CSRIVR; E/LSRE, ratio of E to LSRE; E/LSRIVR, ratio of E to LSRIVR; IVR, isovolumic relaxation; LS, absolute value of peak longitudinal strain; SD, standard deviation; STE, speckle-tracking echocardiography.

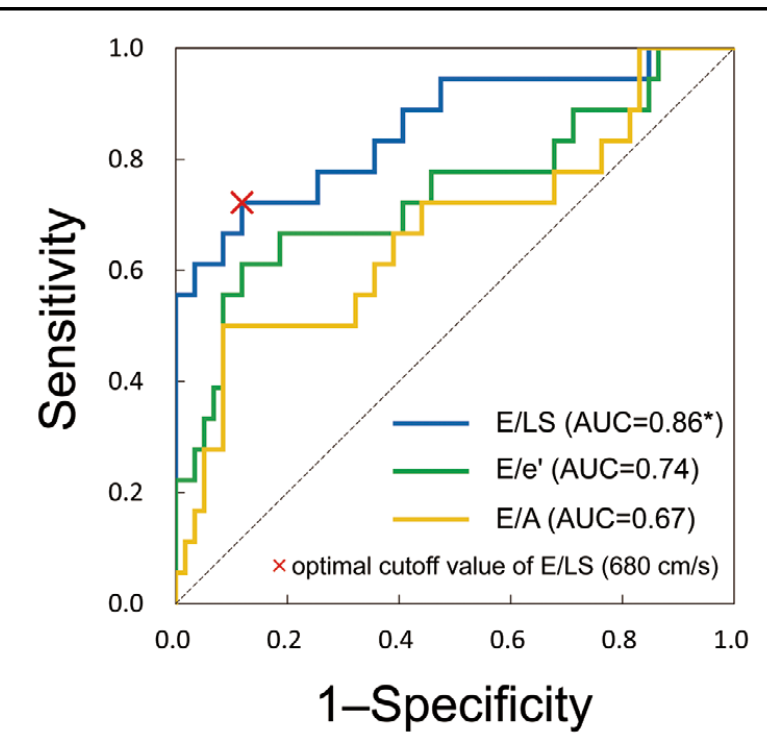

Figure 3. Receiver-operating characteristic curves for E/LS, E/e', and E/A for distinguishing patients with elevated left ventricular filling pressure. ${ }^{*} \mathrm{P}<0.05 \mathrm{vs}$. E/A. AUC, area under the curve; $E / A$, ratio of early-diastolic LV inflow velocity (E) to atrial-systolic velocity (A); $e^{\prime}$, early-diastolic mitral annular velocity; E/e', ratio of $E$ to $e^{\prime} ; E / L S$, ratio of $E$ to $L S$; $L S$, absolute value of peak longitudinal strain.

in which LS showed the strongest correlation with $\tau$ (Figure 2B). There was, however, no statistically significant difference between the correlation of $\mathrm{e}^{\prime}$ with $\tau$ and that of LS $(\mathrm{P}=0.21)$.

\section{Correlations Between LVMDP and Echocardiographic Parameters}

There was a significant correlation between LVMDP and E/A as well as between LVMDP and E/e' (Figures 2C,D). The correlations between LVMDP and STE-derived parameters are summarized in Table 3 . Strain rate parameters during the IVR period significantly but weakly correlated with LVMDP. Strain rate parameters in the early-diastolic phase correlated modestly with LVMDP. In contrast, E/LS and E/CS correlated well with LVMDP. E/LS showed the strongest correlation with LVMDP (Figure 2E). The correlation of E/LS was significantly better than that of $\mathrm{E} / \mathrm{A}(\mathrm{P}<0.05)$ and $\mathrm{E} / \mathrm{e}^{\prime}(\mathrm{P}<0.01)$.

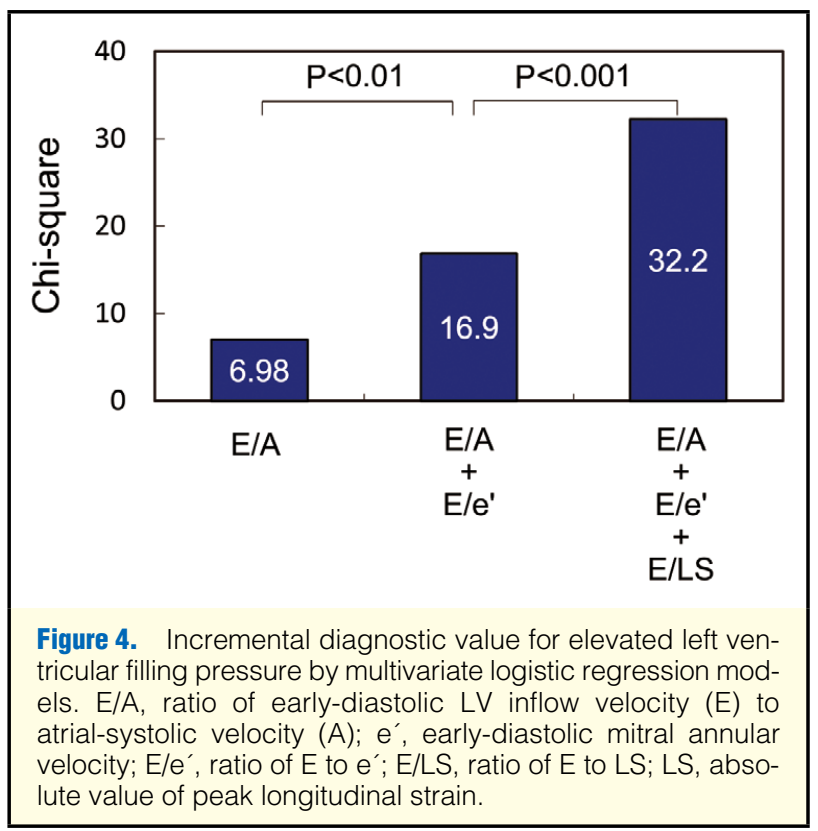

\section{Prediction of Elevated LV Filling Pressure}

The area under the ROC curve of E/LS for distinguishing elevated LV filling pressure was significantly larger than that of E/A, and tended to be larger than that of E/e' (Figure 3). E/ LS distinguished the patients with a sensitivity of $72 \%$ and a specificity of $88 \%$ using the best cutoff value of $680 \mathrm{~cm} / \mathrm{s}$. The additional benefit of E/e' and E/LS to identify patients with elevated LV filling pressure is shown in Figure 4. A significant improvement was obtained by the addition of E/e' $(\mathrm{P}<0.01)$. Also, the addition of $\mathrm{E} / \mathrm{LS}$ improved the diagnostic utility of the model containing $\mathrm{E} / \mathrm{A}$ and $\mathrm{E} / \mathrm{e}^{\prime}(\mathrm{P}<0.001)$.

\section{Analysis in Subgroups According to LVEF}

The $\mathrm{e}^{\prime}$ did not significantly correlate with $\tau(\mathrm{r}=-0.12, \mathrm{P}=0.52)$ in the subgroup with reduced LVEF ( $n=30$, LVEF: $39 \pm 8 \%$ ), whereas $\mathrm{e}^{\prime}$ significantly, but weakly, correlated with $\tau(\mathrm{r}=-0.32$, $\mathrm{P}<0.05$ ) in the subgroup with preserved LVEF ( $\mathrm{n}=47, \mathrm{LVEF}$ : $70 \pm 9 \%$ ). LS significantly correlated with $\tau$ in both subgroups with reduced LVEF $(\mathrm{r}=-0.40, \mathrm{P}<0.05)$ and with preserved LVEF ( $r=-0.32, \mathrm{P}<0.05)$. In both subgroups with reduced LVEF and with preserved LVEF, there were modest correlations between LVMDP and E/A $(r=0.58, \mathrm{P}<0.01$; $\mathrm{r}=0.49$, 

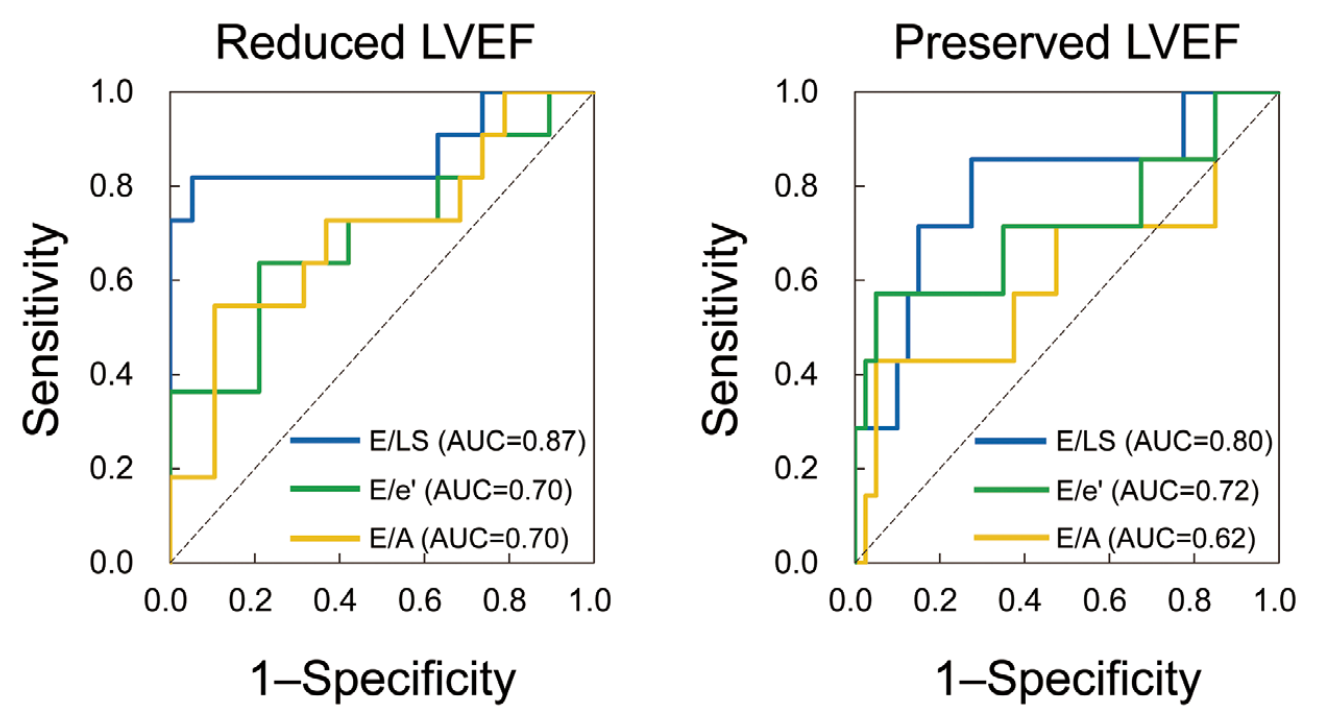

Figure 5. Receiver-operating characteristic curves for E/LS, E/e', and E/A for distinguishing patients with elevated LV filling pressure in patients with reduced LVEF and those with preserved LVEF. AUC, area under the curve; E/A, ratio of early-diastolic LV inflow velocity (E) to atrial-systolic velocity (A); e', early-diastolic mitral annular velocity; E/e', ratio of E to e'; E/LS, ratio of E to LS; LS, absolute value of peak longitudinal strain; LVEF, left ventricular ejection fraction.

$\mathrm{P}<0.001)$ and between LVMDP and $\mathrm{E} / \mathrm{e}^{\prime}(\mathrm{r}=0.39, \mathrm{P}<0.05$; $\mathrm{r}=0.56, \mathrm{P}<0.001)$. In contrast, E/LS correlated modestly with LVMDP in the preserved LVEF group $(\mathrm{r}=0.55, \mathrm{P}<0.001)$ and correlated strongly in the reduced LVEF group $(r=0.73$, $\mathrm{P}<0.001)$. The ROC curves for distinguishing elevated LV filling pressure are shown in Figure 5. In both groups, the area under the ROC curve for E/LS tended to be larger than those for $\mathrm{E} / \mathrm{A}$ and $\mathrm{E} / \mathrm{e}^{\prime}$.

\section{Reproducibility}

Mean intraobserver differences for LS, LSRE, LSRIVR, CS, CSRE, and CSRIVR were 6\%, 10\%, 17\%, 6\%, $8 \%$, and $20 \%$, respectively. Mean interobserver differences for LS, LSRE, LSRIVR, CS, CSRE, and CSRIVR were 7\%, 13\%, 19\%, 7\%, 11\%, and $23 \%$, respectively.

\section{Discussion}

One of the major findings in the present study was that $\mathrm{e}^{\prime}$ significantly but weakly correlated with $\tau$, and E/e' modestly correlated with LVMDP. E/LS correlated well with LVMDP, which distinguished patients with elevated LV filling pressure better than E/A or E/e'.

\section{Conventional Echocardiographic Parameters}

Several Doppler measurements are currently used to evaluate LV relaxation and filling pressure. Mitral annular velocity is reported to be less affected by loading conditions and better reflects $L V$ relaxation than inflow E/A. ${ }^{19-21} \mathrm{E}$ is influenced by filling pressure as well as inversely altered by changes in relaxation, and correcting $\mathrm{E}$ by $\mathrm{e}^{\prime}$ for the influence of relaxation improves its relation with filling pressure. ${ }^{20} \mathrm{E} / \mathrm{e}^{\prime}$ is thus considered to accurately estimate filling pressure in a broad spectrum of patients with preserved or reduced LVEF. According to the results of several studies, ${ }^{22-24}$ the American Society of Echocardiography recommended not to use $\mathrm{e}^{\prime}$ and E/e' in patients with significant mitral annular calcification, surgical rings, mitral stenosis, prosthetic mitral valves, moderate to severe primary mitral regurgitation, and constrictive pericarditis. ${ }^{1}$ Subsequently, Geske et al found no correlation between mean left atrial pressure and $\mathrm{E} / \mathrm{e}^{\prime}$ in patients with symptomatic hypertrophic cardiomyopathy. ${ }^{2}$ Mullens et al also reported no significant correlation between pulmonary artery wedge pressure and $\mathrm{E} / \mathrm{e}^{\prime}$ in decompensated patients with advanced systolic heart failure. ${ }^{3}$ Based on those results, it is widely believed that taking patient characteristics into consideration is necessary when $\mathrm{e}^{\prime}$ and E/e' are used. Also, almost all studies that validated $\mathrm{e}^{\prime}$ and $\mathrm{E} / \mathrm{e}^{\prime}$ were performed in a single center. Therefore, we assessed these parameters against invasively measured LV relaxation and filling pressure in a multicenter study in which the Doppler echocardiographic measurements were standardized. We examined 77 patients with various cardiac diseases after excluding patients with the known restrictions mentioned earlier. As a result, $\mathrm{e}^{\prime}$ significantly but only very weakly correlated with $\tau$, and E/e' correlated modestly with LVMDP, which differed from the results of some previous studies possibly because of the differences in patients' characteristics. These findings suggest that these parameters should not be used as single quantitative parameters to estimate relaxation and filling pressure.

Estimation of LV Relaxation Using STE-Derived Parameters It was previously considered that strain rate during the IVR period and in the early-diastolic phase correlated with $\tau$. However, in our study LV peak global strain tended to better correlate with $\tau$ than did these early-diastolic parameters. The myocardial wall stores energy in the form of elastic recoil during systole and this energy is released when the myocardium relaxes. ${ }^{25}$ Although $\tau$ is widely accepted as a gold standard measure of LV relaxation, it can be affected by contractile properties. In fact, several researchers reported an inverse correlation between $\tau$ and LV contractility. ${ }^{12,26}$ Contraction 
and relaxation are thus physiologically coupled. Although early-diastolic wall deformation may reflect a pure relaxation property, it can be associated with $\tau$ through contractility more strongly than through relaxation. If so, because contractility has a closer relationship with peak strain, peak global strain could correlate better with $\tau$ than would the early-diastolic parameters.

In addition to the underlying physiology, there may be a methodological reason for the advantage of peak global strain over the strain rate during early diastole. Because STE directly extracts a time-strain curve by tracking the echo-dense speckles frame-by-frame and because strain rate is calculated by differentiating the strain by time, peak global strain is a more robust parameter than strain rate when measured by STE. Although global strain rate during the IVR period is theoretically a suitable marker to estimate LV relaxation, because relaxation and the resulting LV pressure decay occur during the IVR period, ${ }^{6}$ accurate measurement of strain rate during the relatively short duration requires an adequate image with high temporal resolution, which is sometimes difficult to obtain during a clinical examination.

We also consistently found that the strain/strain rate in the longitudinal direction tended to correlate with $\tau$ better than in the circumferential direction. This may have resulted in part from the vulnerability of the longitudinally-orientated inner myocardial fibers in the early stage of diastolic dysfunction. ${ }^{27}$ Ishizu et al demonstrated in an animal study that LS, but not CS, significantly correlated with $\tau$ in the presence of LV hypertrophy induced by hypertension. ${ }^{28}$ Because a large number of our patients had hypertension, not circumferential but longitudinal indices could detect subclinical abnormalities in $\mathrm{LV}$ relaxation.

\section{Estimation of LV Filling Pressure Using STE-Derived Parameters}

In the present study, E/LS correlated with LVMDP most strongly among E/A, E/e', and various STE-derived parameters. As is the case with E/e', the parameter that accurately reflects LV relaxation should be the most suitable denominator for this type of index. Therefore, LS correlated most strongly with $\tau$, and E/LS correlated most strongly with LVMDP. Global LS is now widely used as a prognostic marker of various cardiac diseases, ${ }^{29-31}$ and E/LS can be easily evaluated in routine examinations as a parameter of LV filling pressure.

Together with SRIVR as a parameter of LV relaxation, E/ SRIVR has been reported to be an accurate marker of LV filling pressure. ${ }^{6}$ However, we did not find strong correlations between the LVMDP and any E/SRIVR measurements. In addition to the difficulty of accurately measuring SRIVR, the low absolute value of SRIVR is unsuitable as a denominator for indices such as E/e'. SRIVR values were considerably low and it was necessary to correct the denominator of E/SRIVR in patients with extremely low SRivr values as mentioned in the Methods section. Therefore, the instability of E/SRIVR as an index would have decreased the correlations between LVMDP and E/SRIVR.

\section{Influence of LVEF on the Correlation Between LV Filling Pressure and STE-Derived Parameters}

Because of the problem of pseudonormalization of LV inflow, $\mathrm{E} / \mathrm{A}$ is the recommended indicator of elevated LV filling pressure only in patients with reduced LVEF, but not preserved LVEF. ${ }^{1}$ In contrast, E/e' is considered a surrogate marker of LV filling pressure in patients with preserved LVEF. ${ }^{1}$ Our results showed that the diagnostic ability of E/LS for distin- guishing elevated LV filling pressure tended to be superior to $\mathrm{E} / \mathrm{A}$ and E/e' in both groups. Therefore, E/LS could be a useful parameter for $\mathrm{LV}$ filling pressure regardless of LVEF.

\section{Study Limitations and Clinical Implications}

There are several limitations to be acknowledged in this study. First, echocardiographic studies were not performed simultaneously with cardiac catheterization, which may have created differences in loading conditions. However, a previous study showed little difference in the results of the subgroup simultaneously measured echocardiographic and invasive data, when compared with results for the entire population. ${ }^{2}$ Second, although $49 \%$ of the 77 patients had impaired LV relaxation, only $33 \%$ had heart failure of New York Heart Association class II or more and only $23 \%$ had elevated LV filling pressure, partly because LV pressures were measured after stabilizing loading conditions in most of the heart failure patients, which could have weakened the correlation between filling pressure and the echocardiographic parameters. Therefore, the correlation coefficients for all echocardiographic parameters were relatively low in this study population as compared with previous studies. However, even in such a patient population as in the present study, E/e' modestly correlated whereas E/LS strongly correlated with LV filling pressure, and the correlation of E/LS was statistically significantly better than that of E/e'. Furthermore, the addition of E/LS improved the diagnostic utility of the model containing E/A and E/e' for estimating elevated LV filling pressure. In the clinical setting, echocardiography plays an important role in the estimation of LV filling pressure in patients with slightly elevated LV filling pressure who are suspected to have heart failure, but not in healthy subjects or in patients with obvious lung congestion. Therefore, our results might have important clinical implications in the diagnosis of heart failure in such a patient population. Accordingly, E/LS might have an incremental diagnostic value for estimating LV filling pressure in patients with intermediate filling pressure regardless of LVEF. However, we should avoid estimating the LV filling pressure with too much confidence using a single echocardiographic parameter such as E/e'. A larger study is needed to confirm the feasibility and clinical usefulness of $\mathrm{E} / \mathrm{LS}$ in the future.

\section{Conclusions}

Tissue Doppler-derived e' weakly correlated with $\tau$, whereas STE-derived longitudinal parameters tended to better correlate with $\tau$ than $\mathrm{e}^{\prime}$. Although E/e' only modestly correlated with LVMDP, STE-derived longitudinal parameters, especially E/ LS, strongly correlated with LVMDP, which had an incremental diagnostic value for estimating LV filling pressure.

\section{Acknowledgment}

This research was supported by the Research and Development Committee Program of the Japan Society of Ultrasonics in Medicine.

\section{Disclosures}

None.

\section{References}

1. Nagueh SF, Appleton CP, Gillebert TC, Marino PN, Oh JK, Smiseth $\mathrm{OA}$, et al. Recommendations for the evaluation of left ventricular diastolic function by echocardiography. J Am Soc Echocardiogr 2009; 22: 107-133.

2. Geske JB, Sorajja P, Nishimura RA, Ommen SR. Evaluation of left ventricular filling pressures by Doppler echocardiography in patients with hypertrophic cardiomyopathy: Correlation with direct left atrial 
pressure measurement at cardiac catheterization. Circulation 2007; 116: $2702-2708$

3. Mullens W, Borowski AG, Curtin RJ, Thomas JD, Tang WH. Tissue Doppler imaging in the estimation of intracardiac filling pressure in decompensated patients with advanced systolic heart failure. Circulation 2009; 119: 62-70.

4. Bhella PS, Pacini EL, Prasad A, Hastings JL, Adams-Huet B, Thomas JD, et al. Echocardiographic indices do not reliably track changes in left-sided filling pressure in healthy subjects or patients with heart failure with preserved ejection fraction. Circ Cardiovasc Imaging 2011; 4: 482-489.

5. Dokainish H, Sengupta R, Pillai M, Bobek J, Lakkis N. Usefulness of new diastolic strain and strain rate indexes for the estimation of left ventricular filling pressure. Am J Cardiol 2008; 101: 1504-1509.

6. Wang J, Khoury DS, Thohan V, Torre-Amione G, Nagueh SF. Global diastolic strain rate for the assessment of left ventricular relaxation and filling pressures. Circulation 2007; 115: 1376-1383.

7. Kimura K, Takenaka K, Ebihara A, Okano T, Uno K, Fukuda N, et al. Speckle tracking global strain rate E/E' predicts LV filling pressure more accurately than traditional tissue Doppler E/E'. Echocardiography 2012; 29: 404-410.

8. ten Brinke EA, Bertini M, Klautz RJ, Antoni ML, Holman ER, van de Veire NR, et al. Noninvasive estimation of left ventricular filling pressures in patients with heart failure after surgical ventricular restoration and restrictive mitral annuloplasty. $J$ Thorac Cardiovasc Surg 2010; 140: 807-815.

9. Meluzin J, Spinarova L, Hude P, Krejci J, Podrouzkova H, Pesl M, et al. Estimation of left ventricular filling pressures by speckle tracking echocardiography in patients with idiopathic dilated cardiomyopathy. Eur J Echocardiogr 2011; 12: 11-18.

10. Chen S, Yuan J, Qiao S, Duan F, Zhang J, Wang H. Evaluation of left ventricular diastolic function by global strain rate imaging in patients with obstructive hypertrophic cardiomyopathy: A simultaneous speckle tracking echocardiography and cardiac catheterization study. Echocardiography 2014; 31: 615-622.

11. Kasner M, Gaub R, Sinning D, Westermann D, Steendijk P, Hoffmann W, et al. Global strain rate imaging for the estimation of diastolic function in HFNEF compared with pressure-volume loop analysis. Eur J Echocardiogr 2010; 11: 743-751.

12. Weiss JL, Frederiksen JW, Weisfeldt ML. Hemodynamic determinants of the time-course of fall in canine left ventricular pressure. $J$ Clin Invest 1976; 58: 751-760.

13. Paulus WJ, Tschope C, Sanderson JE, Rusconi C, Flachskampf FA, Rademakers FE, et al. How to diagnose diastolic heart failure: A consensus statement on the diagnosis of heart failure with normal left ventricular ejection fraction by the Heart Failure and Echocardiography Associations of the European Society of Cardiology. Eur Heart J 2007; 28: 2539-2550.

14. Yamamoto K, Nishimura RA, Redfield MM. Assessment of mean left atrial pressure from the left ventricular pressure tracing in patients with cardiomyopathies. Am J Cardiol 1996; 78: 107-110.

15. Ommen SR, Nishimura RA, Appleton CP, Miller FA, Oh JK, Redfield MM, et al. Clinical utility of Doppler echocardiography and tissue Doppler imaging in the estimation of left ventricular filling pressures: A comparative simultaneous Doppler-catheterization study. Circulation 2000; 102: 1788-1794.

16. Lang RM, Bierig M, Devereux RB, Flachskampf FA, Foster E, Pellikka PA, et al. Recommendations for chamber quantification: A report from the American Society of Echocardiography's Guidelines and Standards Committee and the Chamber Quantification Writing Group, developed in conjunction with the European Association of Echocardiography, a branch of the European Society of Cardiology. $J$ Am Soc Echocardiogr 2005; 18: 1440-1463.

17. Cohen J, Cohen P, West SG, Aiken LS. Applied multiple regression/ correlation analysis for the behavioral sciences. 3rd edn. Lawrence Erlbaum Associates, Mahwah, NJ, 2003.

18. Hanley JA, McNeil BJ. The meaning and use of the area under a receiver operating characteristic (ROC) curve. Radiology 1982; 143: $29-36$.

19. Hasegawa H, Little WC, Ohno M, Brucks S, Morimoto A, Cheng $\mathrm{HJ}$, et al. Diastolic mitral annular velocity during the development of heart failure. J Am Coll Cardiol 2003; 41: 1590-1597.

20. Nagueh SF, Middleton KJ, Kopelen HA, Zoghbi WA, Quinones MA. Doppler tissue imaging: A noninvasive technique for evaluation of left ventricular relaxation and estimation of filling pressures. $J \mathrm{Am}$ Coll Cardiol 1997; 30: 1527-1533.

21. Sohn DW, Chai IH, Lee DJ, Kim HC, Kim HS, Oh BH, et al. Assessment of mitral annulus velocity by Doppler tissue imaging in the evaluation of left ventricular diastolic function. $J$ Am Coll Cardiol 1997; 30: 474-480.

22. Diwan A, McCulloch M, Lawrie GM, Reardon MJ, Nagueh SF. Doppler estimation of left ventricular filling pressures in patients with mitral valve disease. Circulation 2005; 111: 3281-3289.

23. Bruch C, Stypmann J, Gradaus R, Breithardt G, Wichter T. Usefulness of tissue Doppler imaging for estimation of filling pressures in patients with primary or secondary pure mitral regurgitation. Am J Cardiol 2004; 93: 324-328.

24. Alraies MC, Kusunose K, Negishi K, Yarmohammadi H, Motoki H, AlJaroudi W, et al. Relation between echocardiographically estimated and invasively measured filling pressures in constrictive pericarditis. Am J Cardiol 2014; 113: 1911-1916.

25. Gilbert JC, Glantz SA. Determinants of left ventricular filling and of the diastolic pressure-volume relation. Circ Res 1989; 64: 827-852.

26. Eichhorn EJ, Willard JE, Alvarez L, Kim AS, Glamann DB, Risser $\mathrm{RC}$, et al. Are contraction and relaxation coupled in patients with and without congestive heart failure? Circulation 1992; 85: 2132-2139.

27. Mizuguchi Y, Oishi Y, Miyoshi H, Iuchi A, Nagase N, Oki T. The functional role of longitudinal, circumferential, and radial myocardial deformation for regulating the early impairment of left ventricular contraction and relaxation in patients with cardiovascular risk factors: A study with two-dimensional strain imaging. $\mathrm{J} \mathrm{Am} \mathrm{Soc}$ Echocardiogr 2008; 21: 1138-1144.

28. Ishizu T, Seo Y, Kameda Y, Kawamura R, Kimura T, Shimojo N, et al. Left ventricular strain and transmural distribution of structural remodeling in hypertensive heart disease. Hypertension 2014; 63: 500-506.

29. Okada K, Yamada S, Iwano H, Nishino H, Nakabachi M, Yokoyama $\mathrm{S}$, et al. Myocardial shortening in 3 orthogonal directions and its transmural variation in patients with nonobstructive hypertrophic cardiomyopathy. Circ J 2015; 79: 2471-2479.

30. Sato K, Seo Y, Ishizu T, Takeuchi M, Izumo M, Suzuki K, et al. Prognostic value of global longitudinal strain in paradoxical lowflow, low-gradient severe aortic stenosis with preserved ejection fraction. Circ J 2014; 78: 2750-2759.

31. Enomoto M, Ishizu T, Seo Y, Yamamoto M, Suzuki H, Shimano H, et al. Subendocardial systolic dysfunction in asymptomatic normotensive diabetic patients. Circ J 2015; 79: 1749-1755. 